\title{
Synchronous Liver Metastases from Colorectal Cancer: State of the Art
}

\author{
Ricardo Jorge Teixeira Martins, José Guilherme Lopes Rodrigues Tralhão
}

Pediatric and Adult Liver Transplant Unit, Coimbra Hospital and Universitary Centre Surgery Department, HBP Unit, Coimbra Hospital and Universitary Centre Surgery III Universitary Clinic, Faculty of Medicine of the University of Coimbra Biophysics Institute, IBILI, Faculty of Medicine of the University of Coimbra Coimbra Institute for Clinical and Biomedical Research (iCBR) area of Environment Genetics and Oncobiology (CIMAGO), Faculty of Medicine of the University of Coimbra
Corresponding author:

Ricardo Martins, MD

Praceta Mota Pinto, 3000-075 Coimbra,

Portugal

Phone: +351 239400400

E-mail: ricardo.martins@chuc.min-saude.pt

\section{ABSTRACT}

Colorectal cancer (CRC) is one of the most common cancers in the world and a leading cause of death. The liver is the dominant metastatic site for patients with CRC, and approximately $50 \%$ of patients develop CRC liver metastases (CRCLM) during the course of their disease. Surgical resection, when combined with chemotherapy, can achieve 5-year overall survival $(O S)$ rates of up to $40-60 \%$ for patients with CRCLM. Despite excellent results, recurrence-free survival rates remain low as many surviving patients eventually experience recurrence. Appropriate patient selection is the key to ensure the best perioperative and long-term oncologic outcomes. Synchronous CRCLM are detected at the same time or before the diagnosis of the primary tumor. In general these patients present with a higher tumor burden, more extensive liver disease, and a more aggressive presentation. The 5 -year OS rate in patients with synchronous CRCLM is around $40 \%$, and specific approaches for the decision-making process are mandatory according to the tumor status and resectability at both organ sites. When appropriate and technically feasible, the simultaneous surgical resection approach to synchronous CRCLM should be preferred as it is safe and effective and provides for substantial financial cost savings. Neoadjuvant chemotherapy should be used with precaution, since it can result in lesions "disappearing" or becoming imaging occult. Moreover, the risk of operative morbidity increases with the number of prior chemotherapy cycles. A short period of conversion chemotherapy is recommended, and multidisciplinary discussions of the treatment strategy should be scheduled every two months. An individualized and patient-tailored multidisciplinary approach is mandatory, when trying to define the best treatment of synchronous CRCLM.

Key words: colorectal cancer, liver metastases, liver surgery, hepatectomy

\section{INTRODUCTION}

Colorectal cancer (CRC) is one of the most common cancers in the world and a leading cause of death (1). In 2018, CRC represented the second most common cancer in Portugal, with 10,270 cases, and the second leading cause of death cancer-related with 4,170 deaths (2).
Received: 31.10 .2019

Accepted: 02.12.2019 
The liver is the dominant metastatic site for patients with CRC, and approximately $50 \%$ of patients develop liver metastases (CRCLM) during the course of their disease; $15 \%$ to $25 \%$ of patients, present with synchronous CRCLM at the time of diagnosis, only a small number of patients can be suitable for upfront surgery (3-6).

Surgical resection, when combined with chemotherapy, can provide the greatest opportunity for cure and long-term survival for patients with CRCLM. The clinical outcomes for patients with metastatic CRC have significantl improved over the last decade $(4,7)$.

Chemotherapy, including molecular targeted agents, for metastatic CRC has greatly improved recently and offers an increased chance of conversion hepatectomy for patients with initially unresectable CRCLM. Peri-operative chemotherapy can improve disease-free survival in resected CRCLM patients, and the response to chemotherapy is critical, i.e., the better the tumor responds to systemic treatment the lower the recurrence rate post-resection (7-9).

Multimodal treatment achieves 5-year overall survival (OS) rates of up to $40-60 \%$ for patients with CRCLM. Despite these excellent results, recurrence-free survival remain low and many surviving patients eventually experience disease recurrence. Despite substantial technological improvements with more modern imaging exams, accurate surgical techniques, knowledge of tumor biology and multimodal treatments, about $60 \%$ of patients will still develop recurrence during follow-up, the majority of them within the first two years $(7,10,11)$.

Appropriate patient selection is the key to ensure the best perioperative and long-term oncologic outcomes. An individualized multidisciplinary approach is mandatory for the treatment of patients with CRCLM. The multidisciplinary team should include surgeons with experience in liver and colorectal surgery, mainly for the decision of patients with synchronous presentation $(12,13)$.

The essential factors to determine whether a patient is a candidate for resection, include patient's age and comorbid diseases, tumor location, number and site of CRCLM, extra-hepatic spread, tumor biology and anatomic characteristics, as well as the health care team's experience $(4,7-9,13-15)$.

The risk of recurrence, more aggressive disease and worse prognosis are all associated with advanced T-stage, N+ staging, synchronous metastases, multiple metastases $(>3)$, and bilobar lesions. Obviously, extension of the surgical indication, for initially more aggressive tumors carries a higher risk of disease recurrence $(4,7,16,17)$.

There are different clinical definitions of synchronous CRCLM, although biologically all metastases are synchronous. In our practice, we consider synchronous CRCLM when detected at the time or before the diagnosis of the primary tumor.

In our experience, similar with other published series, patients with synchronous CRCLM present a higher tumor burden, more extensive liver disease (higher number and size of lesions, and bilobar location), and so thus less favorable tumor biology and a more aggressive presentation $(7,16)$.

Five-year OS rate in our series of patients with synchronous CRCLM is $41,1 \%$, comparable with other reported series (Livermet Survey reports a 5-year OS of $39 \%)(6,7)$.

In the clinical setting, the decision-making process for treatment is based on main determinants such as the tumor status and metastatic lesions, the need for emergency surgery for a complicated primary tumor, as well as, the complete resectability of both primary and metastatic lesions.

The surgery for the primary CRC must be done by a specialist colorectal surgeon, being the surgery quality fundamental either for rectum and colon tumors. Specific requisites like total mesocolon/mesorectal excision, lymph node resection and a good margin of resection are required. $(4,6,18,19)$.

Technical resectability is a necessary condition for an appropriate CRCLM resection. Resectability is determined by at least $25 \%$ to $30 \%$ of functional liver tissue, with sufficient inflow (portal vein/hepatic artery) and outflow (hepatic veins). Conversion chemotherapy and several surgical techniques as two stage hepatectomy, associating liver partition and portal vein ligation (ALPPS) and parenchyma-sparing techniques or combined ablation, as well as nonsurgical like portal vein/hepatic vein embolization can improve resectability $(14,15,20-22)$.

Patients with asymptomatic CRC and unresectable synchronous CRCLM, we usually start the treatment with chemotherapy, in order to reach the resectability of CRCLM. After reevaluation if it becomes resectable, the reverse approach (liver first) should be the therapeutic option. For rectal cancer, treatment usually begins with chemotherapy followed by radiotherapy and, then, in the period between irradiation and rectal cancer surgery, CRCLM resection is performed $(4-6,8$, $18,19,23)$.

The advantages of synchronous resections are mainly a decrease in patient anxiety of being subjected to two procedures, two-stage versus a single one, a 
decrease in the overall length of hospital stay, as well as the financial cost. No difference is observed concerning morbimortality in one-stage versus staged procedure $(4,6,12,18,19,24)$. When appropriate and technically feasible, the simultaneous surgery approach to synchronous CRCLM should be preferred as it is safe and effective, and provides for substantial financial cost savings (24).

The treatment adopted for patients that present symptomatic CRC and resectable synchronous CRCLM should comprise the resection of the primary tumor, in case of perforated or occlusive tumors, followed by a short period chemotherapy (3 cycles), and then, surgery for CRCLM. If it becomes resectable, we prefer the reverse approach to surgery (liver first). For rectal cancer, we usually start with chemotherapy, then radiotherapy and, in the window between irradiation and rectal cancer surgery, we perform the resection of the CRCLM (4-6,8,18,19,23).

For patients with symptomatic CRC and resectable synchronous CRCLM, we prefer to adopt resection of the primary tumor for perforated or occlusive tumours, followed by chemotherapy (short period, 3 cycles) and then surgery for CRCLM. However, this is not suitable for tumors with bleeding causing anaemia, for patients treated with transfusions or for those with a good response after chemotherapy $(4-6,8,18,19,23)$.

The resection of the primary tumor, in case of perforated or occlusive tumors, is preferred for patients with symptomatic CRC and non-resectable synchronous CRCLM. This treatment is proceeded by conversion chemotherapy and, then, surgery for CRCLM if, after revaluation the resectability is achieved. $(4-6,8,18$, 19,23).

Neoadjuvant chemotherapy, should be used with precaution, as it can result in "disappearing" lesions or tumors becoming imaging occult. Despite a complete response on imaging, many studies have shown persistent viable tumor cells at the site of the "disappearing lesions". With a lesion based analysis, the incidence of disappearing lesions ranges from $11 \%$ to $36 \%$ of initially detected CRCLM, and half of these lesions can present persistent disease, which may have great impact on patient relapse as well as on survival (25-27).

CRCLM lesions at the greatest risk of disappearing are those $<2 \mathrm{~cm}$ in diameter and $>1 \mathrm{~cm}$ deep in the liver parenchyma, and can be marked with a fiducial marker (coil) before initiation of neoadjuvant chemotherapy $(28,29)$. On other hand, serious liver-related toxicity has been described for irinotecan (steatohepatitis) and oxaliplatin (sinusoidal obstruction). The risk of operative morbidity increases with the number of chemotherapy cycles. Thus, a short period of conversion chemotherapy is recommended, and multidisciplinary discussions of the treatment strategy should be scheduled early, and held every two months. During conversion chemotherapy, information from CT and MRI should be routinely used for restaging. It is crucial to provide information about treatment response (size and morphological criteria), as well as liver function and anatomy, trough the evaluation of steatosis and signs of portal hypertension, as well as the assessment of future liver remnant $(4,6,16,30)$.

\section{IN CONCLUSION,}

"Biology is King, selection of cases is Queen, and the technical details of surgical procedures are the Princes and Princesses....". We need to keep this important principle in mind when trying to define the best treatment of patients with synchronous CRCLM (22).

\section{Funding}

This research received no specific grant from any funding agency in the public, commercial, or notfor-profit sectors.

\section{Conflict of interest statement}

The authors declare no conflict of interest.

\section{REFERENCES}

1. F. Bray, J. Ferlay, I. Soerjomataram, R. L. Siegel, L. A. Torre, and A. Jemal, "Global cancer statistics 2018: GLOBOCAN estimates of incidence and mortality worldwide for 36 cancers in 185 countries," CA. Cancer J. Clin., vol. 68, no. 6, pp. 394-424, 2018.

2. International Agency for Research on Cancer - World Health Organization, "Globocan 2018. Population Fact Sheets: Portugal," vol. 270, pp. 2018-2019, 2018.

3. A. E. M. van der Pool et al., "Trends in incidence, treatment and survival of patients with stage IV colorectal cancer: a population-based series.," Colorectal Dis., vol. 14, no. 1, pp. 56-61, Jan. 2012.

4. F. C. L. Chow and K. S. H. Chok, "Colorectal liver metastases: An update on multidisciplinary approach," World J. Hepatol., vol. 11, no. 2, pp. 150-172, 2019 .

5. D. Moris et al., "Laparoscopic synchronous resection of colorectal cancer and liver metastases: A systematic review," J. Surg. Oncol., vol. 119, no. 1, pp. 30-39, 2019

6. R. Adam et al., "Managing synchronous liver metastases from colorectal cancer: A multidisciplinary international consensus," Cancer Treat. Rev., vol. 41, no. 9, pp. 729-741, 2015.

7. L. Viganò et al., "Early recurrence after liver resection for colorectal metastases: Risk factors, prognosis, and treatment. A LiverMetSurvey-based study of 6,025 patients," Ann. Surg. Oncol., vol. 21, no. 4, pp. 1276-1286, 2014.

8. E. Van Cutsem et al., "ESMO consensus guidelines for the management of patients with metastatic colorectal cancer," Ann. Oncol., vol. 27, no. 8, pp. 1386-1422, 2016. 
9. Y. Maeda, T. Shinohara, A. Nagatsu, N. Futakawa, and T. Hamada, "Long-Term Outcomes of Conversion Hepatectomy for Initially Unresectable Colorectal Liver Metastases," Ann. Surg. Oncol., vol. 23, no. October 2014, pp. 242-248, 2016.

10. R. Adam et al., "The oncosurgery approach to managing liver metastases from colorectal cancer: a multidisciplinary international consensus.," Oncologist, vol. 17, no. 10, pp. 1225-39, 2012.

11. L. Viganò, N. Russolillo, A. Ferrero, S. Langella, E. Sperti, and L. Capussotti, "Evolution of long-term outcome of liver resection for colorectal metastases: analysis of actual 5 -year survival rates over two decades.," Ann. Surg. Oncol., vol. 19, no. 6, pp. 2035-44, Jun. 2012.

12. M. S. Qureshi, P. J. Goldsmith, S. Maslekar, K. R. Prasad, and I. D. Botterill, "Synchronous resection of colorectal cancer and liver metastases: comparative views of colorectal and liver surgeons.," Colorectal Dis., vol. 14, no. 8, pp. e477-85, Aug. 2012.

13. M. A. Choti et al., "Surgical Resection Preferences and Perceptions among Medical Oncologists Treating Liver Metastases from Colorectal Cancer," Ann. Surg. Oncol., vol. 23, no. 2, pp. 375-381, 2016.

14. V. Dhar, R. M. Thomas, and S. A. Ahmad, "Repeat Hepatectomy for Colorectal Liver Metastases," Cancer Treat. Res., vol. 168, no. 1, pp. 203-220, 2016.

15. R. Adam et al., "Repeat hepatectomy for colorectal liver metastases.," Ann. Surg., vol. 225, no. 1, pp. 51-60; discussion 60-2, Jan. 1997.

16. D. Mitchell, Y. Puckett, and Q. N. Nguyen, "Literature Review of Current Management of Colorectal Liver Metastasis," Cureus, vol. 11, no. 1, pp. 1-13, 2019.

17. L. C. Bredt and A. F. Rachid, "Predictors of recurrence after a first hepatectomy for colorectal cancer liver metastases: A retrospective analysis," World J. Surg. Oncol., vol. 12, no. 1, pp. 1-8, 2014.

18. Q. Feng et al., "Timing of hepatectomy for resectable synchronous colorectal liver metastases: For whom simultaneous resection is more suitable - A meta-analysis," PLoS One, vol. 9, no. 8, 2014.

19. Z. Yin et al., "Timing of hepatectomy in resectable synchronous colorectal liver metastases (SCRLM): Simultaneous or delayed?," Hepatology, vol. 57, no. 6, pp. 2346-2357, 2013.
20. A. Andreou, A. Brouquet, E. K. Abdalla, T. A. Aloia, S. A. Curley, and J. N. Vauthey, "Repeat hepatectomy for recurrent colorectal liver metastases is associated with a high survival rate," Hpb, vol. 13, no. 11, pp. 774-782, 2011.

21. K. Neofytou, H. Wasan, and S. Mudan, "Safety of Redo Hepatectomy for Colorectal Liver Metastases after Selective Interarterial Radiation Therapy: A Case Report," Case Rep. Surg., vol. 2014, pp. 1-5, 2014.

22. H. Lang, J. Baumgart, and J. Mittler, "Associating Liver Partition and Portal Vein Ligation for Staged Hepatectomy in the Treatment of Colorectal Liver Metastases: Current Scenario," Dig. Surg., vol. 35, no. 4, pp. 294-302, 2018.

23. P. Pfeiffer, T. Gruenberger, and R. Glynne-Jones, "Synchronous liver metastases in patients with rectal cancer: can we establish which treatment first?," Ther. Adv. Med. Oncol., vol. 10, no. 1, p. 175883591878799, Jan. 2018.

24. A. Ejaz et al., "Synchronous primary colorectal and liver metastasis: Impact of operative approach on clinical outcomes and hospital charges," Hpb, vol. 16, no. 12, pp. 1117-1126, 2014.

25. D. Elias et al., "Evolution of missing colorectal liver metastases following inductive chemotherapy and hepatectomy.," J. Surg. Oncol., vol. 86, no. 1, pp. 4-9, Apr. 2004.

26. J. W. Owen, K. J. Fowler, M. B. Doyle, N. E. Saad, D. C. Linehan, and W. C. Chapman, "Colorectal liver metastases: disappearing lesions in the era of Eovist hepatobiliary magnetic resonance imaging," $\mathrm{Hpb}$, vol. 18, no. 3, pp. 296-303, 2016.

27. K. Tanaka, H. Takakura, K. Takeda, K. Matsuo, Y. Nagano, and I. Endo, "Importance of complete pathologic response to prehepatectomy chemotherapy in treating colorectal cancer metastases.," Ann. Surg., vol. 250, no. 6, pp. 935-42, Dec. 2009.

28. K. Kuhlmann, J. van Hilst, S. Fisher, and G. Poston, "Management of disappearing colorectal liver metastases," Eur. J. Surg. Oncol., vol. 42, no. 12, pp. 1798-1805, 2016.

29. G. Passot et al., "Eradication of Missing Liver Metastases After Fiducial Placement.," J. Gastrointest. Surg., vol. 20, no. 6, pp. 1173-8, 2016.

30. G. Folprecht, "Liver metastases in colorectal cancer," Rev. Oncol., vol. 3, no. 5, pp. 278-279, 2001. 